\title{
Headache after COVID-19 vaccination: updated report from the Italian Medicines Agency database
}

\author{
Camilla Mattiuzzi ${ }^{1} \cdot$ Giuseppe Lippi $^{2}$ (D)
}

Received: 22 May 2021 / Accepted: 27 May 2021 / Published online: 18 June 2021

(c) Fondazione Società Italiana di Neurologia 2021

\section{Dear Editor,}

Recent evidence suggests that headache is common in severe acute respiratory syndrome coronavirus 2 (SARS-CoV-2) infections [1]. Although universal vaccination appears the mainstay for contrasting the ongoing coronavirus disease 2019 (COVID-19) pandemic outbreak, vaccines may generate side effects, including headache and/or migraine [1-3], which shall be timely recognized as adverse reactions. Therefore, this study was aimed to provide an updated analysis on voluntarily reported headache and/or migraine episodes occurred after COVID-19 vaccination in Italy.

An electronic search was carried out in the public Italian Medicines Agency (Agenzia Italiana del Farmaco; AIFA) database of Adverse Drug Reactions (Reazioni Avverse dei Medicinali; RAM) [4], up to May 9, 2021, with the purpose of retrieving information on headache and/or migraine episodes voluntarily reported in Italy after receiving one of the three currently approved COVID-19 vaccines (mRNA nanoparticle-based Comirnaty, Pfizer, New York, NY, USA; mRNA Moderna COVID-19 Vaccine, Moderna, Cambridge, MA, USA; DNA adenovirus-based Vaxzevria, AstraZeneca, Cambridge, UK). For the purpose of this research, the following medicine keywords were used for searching the RAM database: "PFIZER COMIRNATY", "MODERNA COVID19 VACCINE", and "ASTRAZENECA VAXZEVRIA". The three similar reported symptoms headache, migraine, and head pain were grouped into a single entry (i.e., headache) for further analysis. The epidemiologic information on headache disorders in Italy was retrieved from the GHDx database, maintained by the Institute for Health Metrics and

Giuseppe Lippi

giuseppe.lippi@univr.it

1 Service of Clinical Governance, Provincial Agency for Social and Sanitary Services, Trento, Italy

2 Section of Clinical Biochemistry, University of Verona, Piazzale L.A. Scuro, 10, 37134 Verona, Italy
Evaluation [5]. This repository is the most comprehensive worldwide catalog of health-related data. The electronic search was conducted for "headache disorders," with search criteria set to Italy (country), both sexes (sex), all ages (age), and frequency per 100,000 (metric). The statistical analysis was performed with MedCalc Statistical Software (MedCalc Software Ltd., Ostend, Belgium) and MetaXL software (EpiGear International Pty Ltd., Sunrise Beach, Australia). The study was based on free electronic searches of public AIFA and GHDx repositories, so that no ethics committee approval was necessary.

The cumulative frequency of headache disorders in Italy was 13,417 per 100,000 in the last GHDx searchable year (i.e., 2019), thus accounting for a mean frequency of 37 daily cases per 100,000. The rate of headache/migraine episodes voluntarily reported by recipients of COVID-19 vaccines up to May 9, 2021, was 129, 103, and 21 per 100,000 for AstraZeneca, Pfizer, and Moderna vaccines, respectively. The risk of developing headache/migraine episodes was hence the highest for recipients of AstraZeneca vaccine (odds ratio, 3.50; 95\% CI, 3.12-3.93; $P<0.001$ ), followed by those receiving Pfizer vaccine (odds ratio, 2.78; 95\% CI, 2.47-3.13; $P<0.001$ ), whilst the number of voluntary reports for Moderna COVID-19 vaccine was even lower than the daily frequency of headache disorders in Italy (odds ratio, 0.58; 95\% CI, 0.49-0.68; $P<0.001$ ) (Fig. 1). The cumulative rate of headache/migraine episodes after receiving all COVID-19 vaccines was 2.25 -fold higher than the daily frequency of headache disorders (odds ratio, 2.25; 95\% CI, 0.83-6.11).

This analysis of voluntary reports from the Italian Medicines Agency database shows that the headache/migraine episodes may frequently accompany COVID-19 vaccination, and their frequency is especially high in recipients of AstraZeneca and Pfizer vaccines. 
Fig. 1 Odds ratio (OR; and $95 \%$ confidence interval) of voluntarily reported headache/ migraine episodes after receiving COVID-19 vaccination compared to the daily frequency of headache disorders in Italy

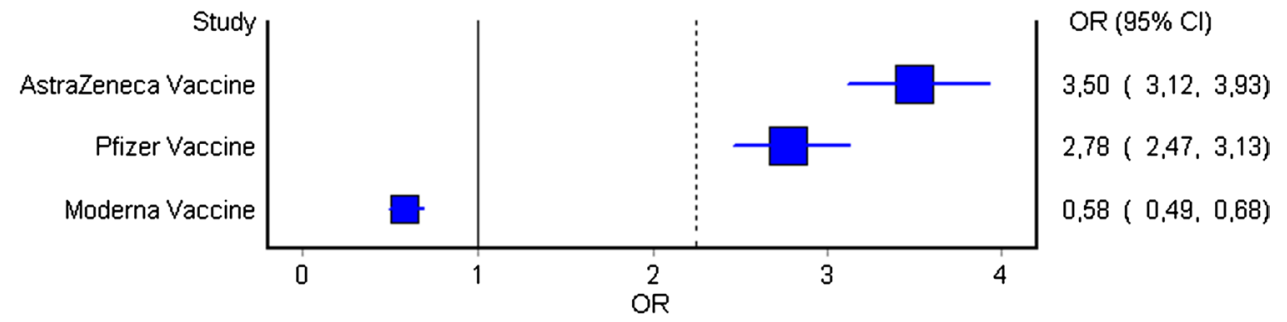

\section{Declarations}

\section{Ethical approval None.}

Informed consent This study does not involve humans.

\section{Conflict of interest None.}

\section{References}

1. Haghdoost F, Carcel C, Chandrasekhar D, Rodgers A, Delcourt C (2021) The impact of Coronavirus disease 2019 (COVID-19) pandemic on migraine disorder. J Neurol 5:1-7. https://doi.org/ 10.1007/s00415-021-10590-x
2. Hervé C, Laupèze B, Del Giudice G, Didierlaurent AM, Tavares Da Silva F (2019) The how's and what's of vaccine reactogenicity. NPJ Vaccines 4:39

3. Baay M, Bollaerts K, Verstraeten T (2018) A systematic review and meta-analysis on the safety of newly adjuvanted vaccines among older adults. Vaccine 36:4207-4214

4. Agenzia Italiana del Farmaco. Reazioni Avverse dei Medicinali. Available at: https://www.aifa.gov.it/sistema-ram. Last accessed, May 20, 2021

5. Global Health Data Exchange. Available at: http://ghdx.healt hdata.org/gbd-results-tool. Last accessed, May 20, 2021

Publisher's note Springer Nature remains neutral with regard to jurisdictional claims in published maps and institutional affiliations. 